\title{
la educaeión en colombia: Periodo de la regeneración
}

\author{
Martha Isabel Bartero \\ Docente Programa de Psicopedagogia \\ Facultad de Educación \\ mbarrero65@gmail.com
}

\section{RESUMEN}

$\mathrm{E}$

ste escrito hace referencia a un periodo histórico de Colombia comprendido entre los años 1886-1899, llamado la Regeneración, gobernado por el partido conservador. Muestra la manera como el gobierno Nacional implementa políticas educativas dirigidas a la conservación del nuevo orden, retomando las teorias de Pestalozzi y la Tomista como criterios pedagógicos, las cuales en el momento de ser llevados al contexto real, se vieron fuertemente influenciados por las condiciones históricas. En este caso este escrito trata de hacer una aproximación a la manera como fueron apropiadas estas teorias a nivel nacional y su repercusión en el Departamento del Tolima.

PALABRAS CLAVES: Pestalozzi, Pedagogia, Historia de las Prácticas Pedagógicas, Educación en la Regeneración

\section{ABSTRACT}

This article reports a study about historical period in Colombia between 1886 and 1899 . This period was called "The Regeneration" and it was governed by the conservative party. It showed the way the National government implemented educational policies aimed at the conservation of the new order. Those polices

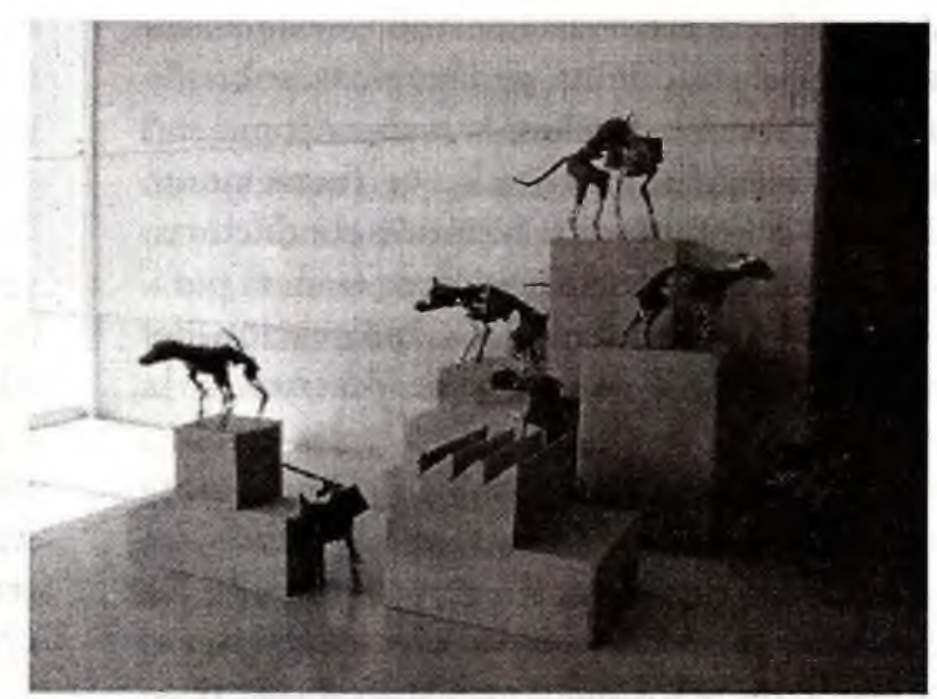

MIGUEL KUAN : "PERRO IDOS..." INSTALACION 2009 - Pitalito

were strongly influenced by Pestalozzi's pedagogical theory and the "Tomista" philosophical trends.

The purpose of the present study is to approach the way those theories were adapted according to the national historical context and its repercussion in the educational policies in the department of Tolima.

KEY WORDS: Pestalozzi, Pedagogy, History of the Practice Pedagogy, Education in the Regeneration Period

Para entender el proceso de formación de una sociedad, es necesario hacer un análisis de la educación, ya que este es un elemento fundamental que ayuda a comprender la forma 
como se consolida la opinión pública de un país y como se mantienen o transforman sus desigualdades y privilegios sociales. La manera como se oriente un tipo particular de educación influye indiscutiblemente en la cultura, el sistema de pensamiento y de mentalidad de un pueblo, por ello se hace necesario tener en cuenta el significado que nos proporciona su conocimiento histórico para tratar de intuir cono ha sido, pero también cuáles son sus metas probables.'

Aunque la educación ha impartido lineamientos a través de modelos pedagógicos sobre la formación del individuo, la manera como son aplicados en la práctica se ve fuertemente influenciada por fuerzas históricas conductoras, que hacen dificil su aplicación de manera pura, dando como resultado una apropiación del modelo, por ello, para acercarnos a la comprensión de la educación, sería erróneo separarla de estas fuerzas. Es importante entonces, tener en cuenta que la educación adopta diversas formas según los lugares, los tiempos, las funciones sociales otorgadas al aprendizaje, y los diferentes grupos que tiene acceso a esas oportunidades.

Partiendo de lo anterior, y para intentar establecer la manera como fue asumida la apropiación del modelo pedagógico pestalozziano en Colombia junto con los efectos que tuvo este en la región del Tolima entre los años de 1886 a 1889 , se requiere por tanto abordar el contexto histórico que lo moldeó, para poder entender el quehacer educativo, el ideal de hombre que se quería formar y el papel que desempeñó el modelo para este propósito.

El periodo de 1886 a 1899 políticamente se caracterizó como el de la Regeneración, hace alusión a una etapa de la vida nacional y regional bastante convulsionada, donde la lucha por el poder y el establecimiento de una carta política que rigiera la vida de los ciudadanos colombianos era una prioridad, en estos momentos el general Rafael Núñez trazaba sus lineamientos que vendrían a materializarse con la Constitución de 1886.

La importancia de estudiar esta temporalidad, radica en que fue precisamente el momento en que la nación se convirtió en una República unitaria; desapareciendo los estados federados para darle paso a los departamentos y su subdivisión en municipios; al fortalecimiento de los poderes del ejecutivo, a la ampliación del periodo presidencial a seis años, la consolidación de la alianza iglesia -Estado en el concordato del 87, el fortalecimiento del Banco Nacional y la creación de un ejército nacional profesional. Esta constitución buscó, a través de sus politicas legislativas, garantizar "un orden" para el pais a través de la legislación administrativa penal, civil, comercial y minera, dando paso a la creación de organismos, instituciones y empresas que contribuyeran a consolidar el nuevo Estado; determinando de esta manera los derroteros sobre la concepción y organización de la Nación, además del papel que debería desempeñar el gobierno y sus entes administrativos frente a la dificil situación económica producida por la caída vertical de las exportaciones, la ruina fiscal, inestabilidad política, los levantamientos y guerras civiles. ${ }^{2}$

Este fue un proyecto que representó para quienes lo promulgaron, "la salvación nacional", el plan global de gobierno incluía tres aspectos: económico, jurídico - político eideológico. ${ }^{3} \mathrm{Se}$

I S!LVA, Renán. La Educación en Colombia 1880 -1930. En: Nueva Historia de Colombia. Editorial Planeta. Bogotá, 1989. P. 61.

'JARAMILLO I IRIBE, Jaime. El proceso de Educación en ta República. 1830 - 1886. En: Historia de Colombia. Editorial Planeta. Tono 2. Bugotá, 1989. P. 233 - 234

'TIRADO MEJLA, Alvaro. Estado y la Politica en el siglo XIX. En: Nueva Historia de Colombia. Editorial Tono 2. Planeta. Bogorá, 1989. P. 176. 
esperaba que su aplicación garantizara el orden, la paz y la seguridad, para permitir la llegada de la prosperidad, la cual ayudaria a enfrenar el cúmulo inmenso de problemas a los cuales el país se veia expuesto.

Para contribuir a este cambio se consideraba que la sociedad no podía existir sin un principio moral unificado, y por ello era solicitada con urgencia la predicación católica del evangelio que permitiría pacificar los ánimos, y establecer un verdadero orden social, donde cada individuo tuviera un rol específico que cumplir y estuviera siempre dispuesto a obedecer y contribuir al cambio, fortaleciendo a la Nación con su trabajo y entrega. La transformación para la unificación nacional, debería realizarse "desde arriba" y se excluía por completo cualquier participación popular, la república debía asumir en este caso, una actitud autoritaria para evitar cualquier tipo de desorden.

Frente a ello, la educación del momento tenia que sufrir indudablemente alteraciones muy profundas; pues el sistema mutuo o método lancasteriano, traido desde 1822 al país, no permitía hacer distingos respecto al credo de sus alumnos, aspecto que le habia generado a la iglesia católica la pérdida de su hegemonia y por ende la unidad moral. Este sistema además, por razones de economía y política habia servido como estrategia para alfabetizar a una masa pobre y ruda, a los hijos de los artesanos y a los campesinos recién llegados a las ciudades, brindándoles una educación elemental de hábitos, virtudes y trabajo, junto con principios básicos de conocimientos que les permitieran asumir una vida civilizada. Debido a ello, todas las clases conformaban una escuela única siendo abolido el concepto de un salón para cada maestro y donde los alumnos más adelantados que aún se encontraban en formación, enseñaban a otros; basándose en la memoria, la repetición, la disciplina y el orden. El éxito de la disciplina se veia aqui reflejado en el hecho de que cada alumno cumplía con el papel de agente vigilante.

Aunque el sistema mutuo se oponia al castigo corporal y en lugar de ello proponia la utilización de recompensan positiva, es decir premios materiales en dinero con el fin de enseñar el valor al trabajo, la connotación de recompensa y castigo fue asumida de forma distinta en nuestro pais, que sometió al niño a castigos físicos, palmetazos, varazos o fuetazos, a sentarse en un banco separado de sus compañeros, entre otros. Estas acciones no eran consideradas como actos salvajes de violencia, de venganza individual, o incluso de represión institucional, sino que estaban ligadas con los fines sociales asignados al hechos de mantener agrupados de modo regular a unos niños fuera de su hogar para la formación de hábitos de obediencia, disciplina y trabajo, es decir el castigo físico contribuia a encauzar la naturaleza humana infantil según el propósito de la sociedad moderna.

La imposibilidad e incapacidad de nuestros dirigentes de crear un modelo propio, capaz de reemplazar el método Lancasteriano, hizo que se adoptase otro extranjero, el Pestalozziano ${ }^{4}$, que utilizado a través del proyecto político regeneracionista, permitiera el mejoramiento de la situación del país, apuntara a la búsqueda de la "salvación nacional" y contribuyera a la erradicación de la memoria, el verbalismo y los castigos físicos; los cuales se habian convertido en los grandes problemas pedagógicos que entorpecian la instrucción de los niños colombianos, impidiéndoles alcanzar un

\footnotetext{
4 Acerca del modelo pedagógico pestalozziano, este fue creado por el suizo Johann Heinrich Pestalozzi (1746 - 1827), refornador de la educación, cuyas teorias establecieron lis cinientus para la noderna educación elemental. Pestalozzi defendia la individualidad del nin̄o y la necesidad de que los maestros fueran preparados para lograr un desarrollo integral del alumno nás que para implantarles conocimientos. Sus ideas ejercieron gran influencia en los sistemas de la escuela elemental del mundo occidental, particularnente en el area de la formación de los profesores.
} 
verdadero desarrollo del entendimiento. Este modelo fue introducido en Colombia bajo el auspicio del presidente conservador Mariano Ospina Rodríguez, y aplicado por José Maria Triana en la Escuela Normal Lancasteriana de Bogotá entre 1845 y 1847 , con el fin de superar la enseñanza mecánica en las clases de gramática y aritmética. Desde 1872, la pedagogía objetiva como fue también llamada, empezó a ser difundida oficialmente en las Instituciones Normales establecidas en cada una de las capitales de los Estados Unidos de Colombia a cargo de los maestros protestantes de la primera Misión Pedagógica Alemana, traídos al pais por los gobernantes liberales radicales. Más adelante, durante el periodo presidencial de Miguel Antonio Caro (1892-1898) el modelo es readaptado buscando cambiar los principios establecidos en las escuelas protestantes y laicas y fue proclamado como oficial, convirtiéndose en la base de la pedagogia para la instrucción primaria colombiana que vino a orientar la educación hasta principios de los años treinta del siglo XX . ${ }^{5}$

En teoria el sistema pestalozziano sugeria que las explicaciones debian basarse en el uso de los objetos mismos de la enseñanza y no en sus representaciones. Lo innovador de su idea de educación elemental, "es su teoría de la educación intelectual, concretada en su famoso método de enseñanza. El punto de partida es la intuición, por la cual los objetos que circundan al niño y que éste percibe llegan así directamente a su conciencia. Esta es la única via de entrada de conocimientos al niño, tal como ya había afirmado Comenio, no valen, pues, los conocimientos transmitidos de un modo verbal o libresco, porque no son conocimientos vivos ni auténticos. Para Pestalozzi el hombre no puede sentir, ni hablar, ni pensar, ni actuar sobre nada que antes no le haya llegado a la conciencia de un modo natural por la intuición" . ${ }^{6}$

Concebia al sujeto como tripartito, es decir un individuo con facultades físicas, morales e intelectuales, por ello las fuerzas que se debían despertar en el niño para encaminarlo concretamente hacia el apetito del bien eran las del corazón (sentimientos), la mente (intelecto) y la mano (gusto); al integrarse conjuntamente estos tres elementos se determinaban nuestros hábitos virtuosos, pero para lograrlo habia que educar equilibradamente cada uno de ellos, teniendo en cuenta la función que desempeñaban en el sujeto.

Es así como en la educación de los sentimientos, debia tenerse en cuenta que el niño antes de pensar y actuar, amaba y creía y estos aspectos debian ser brindados por el padre y la madre para que fueran revertidos por el a los demás; la mente poseia el valor fundamental de la claridad cognoscitiva basada en la experiencia, es decir en la intuición efectiva de las cosas; la intuición de la naturaleza era el fundamento propio y verdadero de la instrucción humana, ésta articulada con sus elementos hacía surgir por una parte las enseñanzas conectadas con la forma (dibujo, geometria), por la otra las conexas con el número (aritmética), y todo lo que se relaciona con los nombres (el aprendizaje lingüistico). El entendimiento tenía como función la representación y la reducción a la unidad, es decir a una idea, que después se desarrollaría poco a poco de manera que fuera más clara? ${ }^{7}$ Por último la tercera fuerza representada en la mano o el arte, era la educación artistica en sentido puramente artesanal o de trabajos manuales que podia ser encausada a trabajos estéticos.

'SAENZ OBREGON, Javier, SALDARRIAGA, Oscar y OSPINA, Amando. Mirar la infancia: pedagogia, moral y modernidad en Colonbia, 1903 - 1946 . Ediciones Uniandes, Editorial I nniversidad de Antiocjuia, Ediciones foro nacional por Colombia y Colciencias. Vol. 1. Santafé de Bogotá, 1997. P. 16-20

"PESTALOZZI, Johann Heinrich. El Canto del Cisne. Introducción, Traducción y Noras por José Maria Quintana Cabanas. Edirorial Laertes Psicopedagogia. España, 2003 . P.24. ${ }^{7}$ ABBAGNANO, N. y VISALBERGHI, A. Historia de la Pedagogia. Fondo de Cultura Econónica. México, 1995. P. 471 - 475. 
Estas tres fuerzas debian ir acompañadas por la noción de infancia o de naturaleza infantil, como una etapa donde debe tenerse cuidado, y conocimiento de las tendencias, inclinaciones y apetencias positivas y negativas del hombre que están en potencia por el cuidado al educar siempre a la evolución mental, ese proceso que va desde lo perceptible por los sentidos, lo singular y lo inmediato, hacia lo abstracto, lo universal y lo inaprensible por los sentidos y por último por la necesidad de simpatia en el maestro y de alegría en todo el proceso de educación del niño en la escuela.

Para el pestalozzianismo era preciso conocer la importancia de la infancia como la etapa en la cual se podia detectar tendencias e inclinaciones tanto positivas y negativas, para partir de ellas y empezar a educar bajo el cuidado de la evolución mental el niño; por eso el papel que jugaba el maestro era fundamental ya que utilizando su alegria y simpatía, evitando el castigo físico, podía educar íntegramente.

Aunque el método no negaba la importancia de la memoria, de hacer leer, escribir y repetir a un niño, consideraba que era más importante enseñarle a pensar. Se podía aprovechar las opiniones de los demás y sacar alguna ventaja del hecho de conocerlas; pero se podía además, hacer que el niño fuera útil para las otras personas mediante el trabajo de su propio entendimiento, de sus propias investigaciones. Sólo así era posible que el individuo fuera acreedor al derecho de ser miembro valioso de la sociedad. ${ }^{8}$
Este modelo fue tomado y adaptado por el Estado, pero no fue seguido al pie de la letra, sino que por el contrario, se obviaron aspectos que se consideraron peligrosos, pues el niño podía quedarse solo, "viendo las cosas". En el terreno de los saberes, el sistema fue asumido como una forma de enseñanza en las escuelas, evadiendo la fundamentación en la producción de la ciencia, basándose solo en desarrollar los sentidos del niño.

A nivel de estrategias político - religiosas, para eliminar el riesgo de formar nin̄os materialistas, se revaloró la tradición científica y catequista religiosa, asumiendo la filosofia neoescolástica para la modernización del sistema escolar. Los avances metodológicos y organizativos de los saberes experimentales fueron usados pero asegurando los fines sobrenaturales y morales para el individuo, la sociedad y el Estado, es decir fue aceptado el discurso moderno, pero a la vez se validó la lectura de la palabra de Dios.

La regeneración propició un ambiente favorable para el auge del neotomismo ${ }^{10}$, buscando la coexistencia entre el pensamiento moderno sin perder la creencia en Dios, manteniendo de esta manera el horizonte católico; para ello se valió de la encíclica Aeterni Patris $^{11}$ de León XIII:

"...así también al presente debe esperarse principalisimamente del omnipotente poder de Dios y de su auxilio, que las inteligencias de los hombres, disipadas las tinieblas del error, vuelvan a la verdad. Pero no se han de despreciar

\footnotetext{
"PESTALOZZ1, Johamn Heinrich. Cartas sobre Educación Infantil. Clásicos del Pensamiento. Madrid: Editorial Tecnos, 1988. P. 79-83

"SAENZ OBREGON, Javier, SALDARRLAGA, Oscary OSPINA, Amando. Mirar la infancia: pedagogia, moral y modernidad en Colonbia, 1903 - 1946 . Ediciones Uniandes, Editorial Universidad de Antioquia, Ediciones foro nacional por Colombia y Colciencias. Vol. 1. Santafé de Bogotá, 1997. P. 83-85.

${ }^{10}$ A cerca del neotonismo, este fue un sistena legado de Santo Toniás, que mediaba por las relaciones entre fe y razón. La razón no podia denıostrar todo lo que es de percinencia de la fe o la fe misma perderia todo mérito pero podia servir a la fe. Su principio básico era que razón y fe trabajan juntas, aun existiendo diferencias entre anıbas. La razón denustraba algumas verdades de la fe, como por ejemplo la existencia y la unidad de Dios, los nisterios de la fe a través de intágenes, netáforas y similitudes, y respondia a las djjeciones de los ateos. Razón y fe eran consideradas dos disciplinas diferentes, pero compartian la misna escala jerárquica.

"León XIII Epistola enciclica Aeterni Patris Sobre la restauración de la filosofia cristiana, conforme a la Doctrina de Santo Tomás de Aquino. 4 de agosto de 1879. A cetca del Aeterni Patris, el Papa León XIII, expuso su convicción de que la filosofia moderna subjetivista estaba en la raiz de los problemas sociales y politicos en la enciclica Aeterni Patris (1879), que abogaba por el restablecimiento de la filosofia de Santo Tomás de Aquino como base de la renovación social y politica, y a la que siguieron ochenta y ocho declaraciones sobre teoria y práctica politica, y la enciclica sobre la condición del trabajo, Rerun Novarum (1891). Esta encíclica sostenia que la propiedad privada era un derecho natural, dentro de los limites de la justicia, pero condenaba al capitalismo como causa de la pubreza y degradación de nuchos trabajadores.
} 
ni posponer los auxilios naturales, que por beneficio de la divina sabiduría, que dispone fuerte y suavemente todas las cosas, están a disposición del género humano, entre cuyos auxilios consta ser el principal el recto uso de la filosofia. No en vano imprimió Dios en la mente humana la luz de la razón, y dista tanto de apagar o disminuir la añadida luz de la fe la virtud de la inteligencia, que antes bien la perfecciona, y aumentadas sus fuerzas, la hace hábil para mayores empresas. Pide, pues, el orden de la misma Providencia, que se pida apoyo aun a la ciencia humana...

...pertenece a las ciencias filosóficas, defender religiosamente las verdades enseñadas por revelación y resistir a los que se atrevan a impugnarlas. Bajo este respecto es grande alabanza de la filosofia el ser considerada baluarte de la fe y como firme defensa de la religión..."12

En Colombia fue Monseñor Rafael María Carrasquilla quien lideró la ideologia de León XIII; para Carrasquilla, la ciencia debia estar subordinada a la religión, toda ley venía de Dios y no era posible aceptar otro origen.

Es en el gobierno de Miguel Antonio Caro (1892-1898) que se instaura el neotomismo, fundamentalmente en la versión de Jaime Balmes filósofo y sacerdote español quien a través de sus escritos defendió el papel civilizador del catolicismo en la historia de occidente, analizando el problema de la verdad y de la evidencia, oponiéndose a la tradición del empirismo británico y del idealismo alemán. Para Caro, las enseñanzas del benthamismo eran contrarias al espíritu de la doctrina cristiana, cuando aseguraban que la ética tenía que construir una ciencia positiva reguladora de la conducta humana, partiendo del análisis de lo dado y puesto en la vida humana. ${ }^{13}$ Esta discusión fue seguida por Marco Fidel Suárez ${ }^{14}$, quien refutó el positivismo por considerarlo como materialista y ateo, ya que reconocia como válido solo aquello que partia de la experiencia y del conocimiento empirico de los fenómenos naturales, motivo por el cual la metafisica y la teologia eran consideradas como sistemas de conocimientos imperfectos e inadecuados, sugiriendo el abandono de la especulación de lo sobrenatural a favor de la investigación cientifica.

El gobierno regeneracionista a través del concordato firmado con el Vaticano en 1887 le da a la iglesia católica la función de promulgar la práctica de la religión, y sobre todo la intervención en los asuntos de la moral pública y privada de los ciudadanos y de los problemas del control social. Con este acto, la iglesia católica asumió el control del contenido de la enseñanza, ella se encargaría de moldear las tres fuerzas del sujeto: corazón, mente y mano, pero partiendo de principios católicos neotomistas utilizando como base la pedagogia pestalozziana.

Bajo esta filosofía se consolidó el cristianismo práctico como proyecto nacional de dominación, trabajo desarrollado por la iglesia, la cual era vista por los dirigentes políticos hegemónicos conservadores como la única institución capaz de establecer una ligadura ético-religiosa eficaz dentro de un país extenso y desarticulado, con extremas desigualdades sociales, raciales y regionales. Esta institución poseia un aparato burocrático centralizado doctrinario y

\footnotetext{
12 LEON XIII. Epistola Enciclica Aeterni Patris. Solore la Restauración de la Filosofia Cristiana, Conforme a la Doctrina de Santo Toniás de Aquuino. 4 de agosto de 1879 1"SIERRA MEJLA, Rubèn. La filosotia en Colombia. En: Nueva Historia de Colombia. Editorial Planeta. Tono IV. Bogotá, 1989. P 212.

${ }^{14}$ Cuardo se posesionó en la Presidencia Miguel Antonio Caro, lo confimó en 1892 como ministro de Relaciones Exceriores SILVA, Renán. La Educación en Colombia
} 1881-1930. En: Nueva Historia de Colonbia. Tomo IV. Edirorial Planeta. Bogotá, 1989. P 70 
organizado que gozaba del respeto de las grandes masas; tenia una larga experiencia educativa desarrollada durante tres siglos de dominación colonial, lo que le habia permitido controlar el aparato educativo; era la única con una propuesta y un saber pedagógico que armonizaban a la perfección con los fines sociales asignados a la educación por el nuevo ordenamiento constitucional, que incluía el tipo de sujeto, hombre y ciudadano que se pretendía formar; y además contaba con recursos materiales y humanos, tanto internos como externos, para colaborar con el proyecto en el plano educativo. ${ }^{15}$

Con el fin de trazar normas de organización para la enseñanza e imponer un sistema de educación nacionalmente unificado, el gobierno regeneracionista a través de la constitución de 1886, declaró que la instrucción primaria costeada con fondos públicos "sería gratuita pero no obligatoria"16, la educación debia ser por tanto, obra tan solo de los particulares, frente a lo cual el papel del Estado era el de actuar alli donde no llegaba o no se interesaba por llevar la iniciativa privada. El Estado como agente educativo, debia funcionar más como complemento, pero debia siempre mantener la disposición de, "la suprema inspección y vigilancia" a través del poder central. Esto permitió que desde entonces, las congregaciones religiosas volvieran a monopolizar la educación, por lo menos hasta más o menos 1930 .

De esta manera el Estado estableció la enseñanza obligatoria de la religión católica, la observancia de las prácticas piadosas, se reservó la facultad de imponer textos de religión, filosofia y moral, de denunciar y hacer excluir a todo docente que pudiera aparecer como sospechosos de creencias morales o religiosas distintas, interferir el contenido literario o científico, se neutralizó la posibilidad de crear un modelo pedagógico local y cesó con la participación de los maestros y los padres en discusiones sobre la educación y la pedagogia, pues era preciso seguir lo establecido. ${ }^{17}$

El modelo pestalozziano quedó totalmente institucionalizado; a través de tres textos oficiales. El primero, fue el tratado -manual elementos de pedagogía de los hermanos Luis y Martín Restrepo Mejia (1885); el segundo, fue un reglamento-manual: el reglamento de escuelas primarias de la república de Colombia conocido como Plan Zerda expedido en 1893, durante el periodo presidencial de Miguel Antonio Caro, el cual se mantuvo casi intacto hasta 1903, y un tercer texto soporte fue creado más adelante, la ley 39 de 1903 conocida como ley Uribe orgánica de Instrucción Pública y su Decreto Reglamentario 491 de $1904 .^{18}$

El plan Zerda de Instrucción, estableció además que la meta del sistema escolar debía ser la unidad nacional y el fomento de la riqueza a través de la formación de obreros cualificados, en lugar de letrados inútiles, formados bajo preceptos morales y religiosos, insistiendo en la educación civica que permitiera despertar tanto en maestros como en estudiantes el amor a la patria, el reconocimiento de sus héroes, de la bandera y del himno nacional. De esta manera la escuela pública primaria quedó definida como esencialmente práctica y encaminada a dotar al alumno de nociones que lo habilitaran para el ejercicio de la ciudadanía y lo prepararan para el trabajo en la industria, la agricultura y el comercio.

\footnotetext{
"s SILVA, Renain. La Educación en Colombia 1881-1930. En: Nueva Historia de Colombia. Tomo IV. Editorial Planeta. Bogotá, 1989. P 70

"Constitución 1886. Titulo III. De los derechos civiles y garantia suciales. Articulo 41

"IARAMILLO URIBE, Jaime. El proceso de Educación en la República. 1830-1886. En: Nueva Historia de Colombia. Editorial Planeta. Tomo Il. Bogotá, 1989. P 234.

in SiLVA, Renán. La Educación en Colombia 1881-1930. En: Nueva Historia de Colonibia. Tonu IV. Editorial Planeta. Bogotá, 1989. P 71.72.
} 
"La educación fue dividida en primaria, secundaria y profesional. Se organizó la inspección educativa y se establecieron las direcciones departamentales de educación, que estarian bajo el control de los gobernadores. Se dejó a cargo de los departamentos la instrucción primaria en lo que se referia al nombramiento y pago de los maestros. A cargo de la Nación quedó el suministro de útiles escolares y por cuenta de los municipios el suministro de locales. Se estatuyeron unas juntas provinciales de educación compuestas por el prefecto de la provincia y 3 ó 4 personas nombradas" . ${ }^{19}$

En el Tolima, fue asumido con mucho entusiasmo el ideario regeneracionista, por parte de los lideres de fuerzas regionales conservadoras quienes criticaron duramente la educación radical liberal, manifestándose en contra de los maestros formados en las Normales federalistas, pidiendo que en su lugar, trajeran a los Hermanos de las escuelas cristianas, quienes brindaban una mejor educación a menor costo, proyectada a infundir los dogmas de la religión católica.

A pesar de esta disposición, en los dos primeros años de establecido el régimen, la escuela tolimense aún no se encausaba definitivamente por los senderos del proyecto católico, hecho que ocasionó que a finales de 1886 se originara un movimiento crítico en pro de una mayor presencia cristiana en toda la organización y en la orientación de la escuela.$^{20}$

En 1886 se habian abierto pocas escuelas debido a que los maestros titulados hasta ese momento en el departamento del Tolima habian sido durante la guerra hostiles al gobierno nacional.
En vista de esta situación el gobernador Casablanca inició trámites para traer confraternidades cristianas que dirigieran escuelas y colegios del Tolima, logrando su cometido solo hasta el año de $1895 .^{21}$

Esta misma labor fue apoyada por el padre Esteban Rojas Tobar, quien con éxito trajo a la provincia del Sur para 1892 a los Hermanos Maristas y las Hermanas de la Caridad; pero el presbítero fue más allá y construyó todo un proyecto educativo, con el cual emprendió una campaña cristianizadora, en compañia de sacerdotes formados para este fin, controlando la educación y excomulgando a todos aquellos que se resistieran a enseñar y recibir educación religiosa.

De acuerdo a la ley, la estructura curricular de los colegios femeninos debía ser orientada a preparar a la mujer para ser educadora, buena esposa, ama de casa y al mismo tiempo propulsora de sanas costumbres bajo una solida formación católica, ya que ella representaba para el proyecto regeneracionista y la pedagogia pestalozziana la base del hogar, la familia y la sociedad; mientras que para los hombres, el currículo debía ayudar a formarlo en el amor al deber, el afecto a la familia, el patriotismo sincero y la afición por el trabajo.22

En las prácticas, las escuelas funcionaron académicamente teniendo en cuenta las normas oficiales, pero su organización y disciplina interna fueron guiados según los criterios de la orden que dirigia la escuela, dando como resultados diferentes formas de apropiación del modelo pestalozziano.

Los planes de estudio sufrieron durante este

\footnotetext{
"IARAMILlO URIBE, Jaine. El pruceso de Educación en la República. 1830-1886. En: Nueva Historia de Colombia. Editorial Planeta. Tomo ll. Bogotá, 1989. P 234.

30 RAMIREZ BAHAMON, Jairo. Historia Social de una Utopia Escolar. La Educación en el Estado Soberano del Tolima 1861-1886. Universidad Surcolombiana. Instituto Huilense de cultura. Fondo de Autores Huilenses. Asociación de Instituciones Huilenses. Acadenia Huilense de Historia. Neiva, 1998. P 318-319.

IRAMIREZ BAHAMON, Jairo. Esplendor y ocaso del proyecto de escuela liberal. Huila siglo XIX. Editorial Universidad Surcolonibiana. Neiva, 2007. P 130.

"RAMIREZ BAHAMON, Jairo. Santa Librada del Siglo XIX. Universidad Surcolombiana -ECOPETRO. Instituto Huilense de cultura. Fondo de Autores Huilenses.

Axsciacion de Instituciones Huilenses. Acadenia Huilense de Historia. Neiva, 1995. P 302.
} 
periodo varios cambios, no fundamentados en la ley, ocasionando deficiencias en las enseñanzas y la no culminación de los estudios por parte de los alumnos; además de elló hubo inconformidades frente a los currículos pues para los caudillos del departamento del Tolima, estos no respondian a las necesidades de la región y no estaban acorde con la situación de pobreza de la mayoria de la población tolimense. Frente a estas dificultades se sumaron otras que contribuyeron en definitiva a opacar el proyecto de expansión católico y de igual manera impidieron el funcionamiento continuo de las escuelas. Por un lado, existieron desacuerdos para decidir el sitio de ubicación de las sedes, no se contó con presupuesto suficiente para construirlas ni para reparar las existian, ya que habian sido usadas como cuarteles durante la guerra ; aunque fueron nombrados 92 maestros para trabajar en las escuelas, muchos de ellos carecian de idoneidad para desempeñar la labor de educador pues además de hacer una mala aplicación del Plan Zerda, continuaron con los golpes de vara y las humillaciones públicas, heredadas del sistema de enseñanza mutua.

En cuanto a lo politico, a pesar del centralismo, el gobierno tenía escaso poder efectivo en cada una de sus provincias para garantizar el cumplimiento de las disposiciones educativas, ya que las estructuras locales y regionales de poder eran las que marcaban de manera cotidiana las prácticas educativas. Por último se encontró la Guerra de los Mil Días iniciada en octubre de 1899 , catástrofe que le dio fin al proyecto católico, acabando con la existencia de los planteles educativos, al ser clausurados.

Se puede apreciar que fueron muchos los factores que incidieron en la forma como fue asumido el modelo pestalozziano a nivel nacional y regional, seria interesante observar la manera como otros modelos hicieron presencia antes y después de este periodo, para tratar de acercarnos a la comprensión del hombre actual, estableciendo como se ha consolidado su pensamiento y sus prácticas culturales, cuales de ellas han desaparecido y cuales han perdurado; entender el proceso de formación de la sociedad actual, a través del análisis de la educación y los objetivos del aprendizaje, teniendo en cuenta por supuesto la normativa social y cultural.

\section{Referentes Bibliográficos}

ABBAGNANO, N. y VISALBERGHI, A. Historia de la Pedagogia. Fondo de Cultura Económica. México, 1995. JARAMILLO URIBE, Jaime. El proceso de Educación en la República. 1830-1886. En: Nueva Historia de Colombia. Editorial Planeta. Tomo II. Bogotá, 1989.

LEON XIII. Epistola enciclica Aeterni Parris. Sobre la Restauración de la Filosofia Cristiana, Conforme a la Doctrina de Santo Tomás de Aquino. 4 de agosto de 1879. PESTALOZZI, Johann Heinrich. Cartas sobre Educación Infantil. Clásicos del Pensamiento. Madrid: Editorial Tecnos, 1988.

PESTALOZZI, Johann Heinrich. El Canto del Cisne. Introducción, Traducción y Notas por José María Quintana Cabanas. Editorial Laertes Psicopedagogia. España, 2003 RAMÍREZ BAHAMÓN, Jairo. Esplendor y ocaso del proyecto de escuela liberal. Huila siglo XIX. Editorial Universidad Surcolombiana. Neiva, 2007. P 130.

RAMÍREZ BAHAMÓN, Jairo. Historia Social de una Utopia Escolar. La Educación en el Estado Soberano del Tolima 1861-1886. Universidad Surcolombiana. Instituto Huilense de cultura. Fondo de Autores Huilenses. Asociación de Instituciones Huilenses. Academia Huilense de Historia. Neiva, 1998. P 318-319.

RAMÍREZ BAHAMÓN, Jairo. Santa Librada del Siglo XIX. Universidad Surcolombiana -ECOPETROL. Instituto Huilense de cultura. Fondo de Autores Huilenses. Asociación de Instituciones Huilenses. Academia Huilense de Historia. Neiva, 1995.

SAENZ OBREGON, Javier, SALDARRIAGA, Oscar y OSPINA, Armando. Mirar la infancia: pedagogia, moral y modernidad en Colombia, 1903 - 1946. Ediciones Uniandes, Editorial Universidad de Antioquia, Ediciones foro nacional por Colombia y Colciencias. Vol. 1. Santafé de Bogotá, 1997.

SIERRA MEJíA, Rubén. La filosofia en Colombia. En: Nueva Historia de Colombia. Editorial Planeta. Tomo IV. Bogotá, 1989. P 212.

SILVA, Renán. La Educación en Colombia 1880 -1930. En: Nueva Historia de Colombia. Editorial Planeta. Bogotá, 1989.

TIRADO MEJIA, Álvaro. Estado y la Política en el siglo XIX. En: Nueva Historia de Colombia. Editorial Tomo 2. Planeta. Bogotá, 1989. 